\title{
A 2.4 GHz GaAs HBT stacked power amplifier with inductance compensation
}

\author{
Tao Chen ${ }^{1 \mathrm{a})}$, Xiaohong Sun ${ }^{1}$, Jianhui $\mathrm{Wu}^{1}, \mathrm{Xu} \mathrm{Niu}^{2}$, \\ and Huai $\mathbf{G a o}^{2}$ \\ ${ }^{1}$ National ASIC System Engineering Research Center, Southeast University, \\ Nanjing 210096, China \\ ${ }^{2}$ RF Power Device and Circuit Engineering Research Center, \\ Suzhou 215123, China \\ a)seu_ct@sina.com
}

\begin{abstract}
A stacked power amplifier (PA) using 2- $\mu \mathrm{m}$ GaAs HBT process is presented for $2.4 \mathrm{GHz}$ application. The stacked configuration can increase the output power by raising the supply voltage of the circuit. Additionally, a novel inter-stage matching technique with series $\mathrm{RC}$ is proposed to suppress the efficiency degradation caused by the parasitic capacitance. The measurement results show the compensation technique improves PAE by more than $6 \%$, while the error vector magnitude (EVM) is enhanced. And the fabricated PA shows a gain of $23.7 \mathrm{~dB}$ and a saturated output power of $32 \mathrm{dBm}$ with PAE of $50 \%$, while the EVMs are lower than $3 \%$ up to $26 \mathrm{dBm}$ of OFDM/64QAM output power.
\end{abstract}

Keywords: heterojunction bipolar transistors (HBTs), power amplifier, stacked structure

Classification: Integrated circuits

\section{References}

[1] M. F. Lei, Z. M. Tsai, K. Y. Lin and H. Wang: IEEE Trans. Microw. Theory Tech. 55 [12] (2007) 2802.

[2] A. K. Ezzeddine and H. C. Huang: IEEE RFIC Symp. (2003) 215.

[3] L. Wu, R. Tao, U. Basaran, J. Luger, I. Dettmann and M. Berroth: 34th European Microwave Conference (2004) 53.

[4] L. Wu and M. Berroth: IEEE Trans. Microw. Theory Tech. 56 [9] (2008) 2040.

[5] C. C. Shen, F. H. Huang, C. K. Lin, H. Y. Chang, Y. J. Chan and Y. C. Wang: Microwave Conference (2008) 1.

[6] T. J. Farmer, A. Darwish and M. E. Zaghloul: IEEE Microw. Wireless Compon. Lett. 20 [5] (2010) 286.

[7] T. J. Farmer, A. Darwish, B. Huebschman, E. Viveiros, H. A. Hung and M. E. Zaghloul: IEEE Microw. Wireless Compon. Lett. 21 [10] (2011) 544. 
[8] A. Agah, H. Dabag, B. Hanafi, P. Asbeck, L. Larson and J. Buckwalter: IEEE RFIC Symp. (2012) 57.

\section{Introduction}

Wireless local area network (WLAN) has been widely used in modern life, especially the $2.4 \mathrm{GHz}$ WLAN based on IEEE $802.11 \mathrm{~g} / \mathrm{n}$ which employs orthogonal frequency division multiplexing (OFDM) modulation for high data rate. OFDM modulation possesses a very high peak-to-average power ratio (PAPR) and requires high linear power amplifier to avoid signal distortion. The linearity can be achieved by setting the amplifier operate at power back off condition. High power and high efficiency PA is demanded for wide area wifi networks.

In order to obtain the maximum output power, rather than for an optimum gain, the device is matched to a load that allows the maximum voltage and current swings. However, the required output matching impedance is usually much lower compared to the system impedance, making the matching circuit design complicated. Stacked power amplifier is an output power enhancement circuit based on voltage multiplication rather than current increasing. Increasing current swing by means of increasing the active devices size or connecting more devices in parallel will decrease the optimum load impedance, and make it difficult to match. The voltage swing increasing technique through connecting the active devices in series can get a higher impedance [1].

There have been reports of the implementations of stacked power amplifier at low frequency $[2,3,4,5,6]$. But at high frequency, the parasitic capacitance effect of an active device becomes obvious. Conventional structure cannot fulfil the inter-stack matching. An inter-stack inductor was adopted in $[7,8]$ to complete the inter-stack matching circuit at high frequency. However, this inductor will occupy a large area and bring some ohmic loss that decreases the output power. In this letter, we present a novel inter-stack matching structure without inter-stack matching inductor at high frequency, which improves PAE and linearity of stacked power amplifier.

\section{Circuit design}

The proposed stacked power amplifier architecture is shown in Fig. 1 using a stack of two GaAs HBT transistors which have the same emitter area. The HBT Q1 collector and Q2 emitter are connected. The base of HBT Q2 is connected to a series resistor $R_{b}$ and capacitor $C_{b}$. The topology of the amplifier allows a maximum collector voltage to be $2 \times B V c e$, where $B V c e$ is the breakdown voltage between collector and emitter of an HBT. The supply voltage $V_{C C}$ is set to $8 \mathrm{~V}$. Both bottom and top transistors of the stacked structure are biased in the same condition. The difference between this structure and classic cascode structure is the inter-stage optimum power 


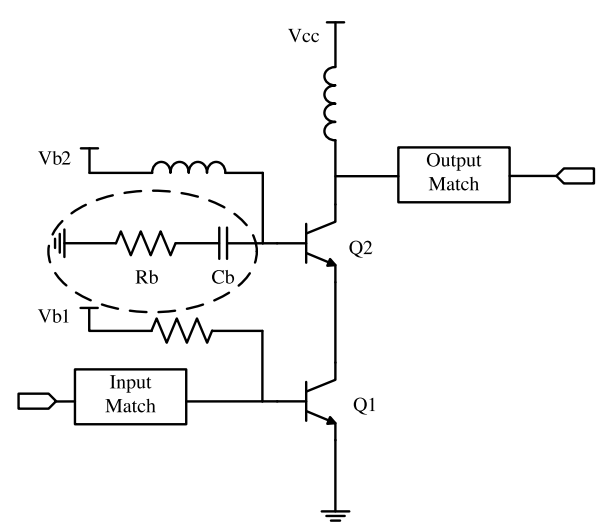

Fig. 1. Schematic of dual stacked power amplifier with series RC at the base of the upper transistors.

match. Unlike in a classic cascode structure where the base of the upper transistors is at RF ground, a relatively small external base capacitance in series with a resistance is introduced to allow an RF swing at the base of the upper transistors. The dual stacked amplifier doubles the voltage swing, and the series devices share the same current swing. To obtain high output power, the output voltage swing and current swing must be large enough.

At low frequency, the input impedance and the optimum load impedance of each device in the stack are purely resistive. The optimum output load impedance of each stacked device is $R_{o p t}=V_{s w g} / I_{s w g}$, where $V_{s w g}$ and $I_{s w g}$ are the maximum voltage and current swing respectively. The optimum load resistance of the dual stacked devices can be assumed to be two times of $R_{\text {opt }}$. The load impedance of the bottom transistors, which is the input impedance of the top transistors, can be adjusted through altering the value of capacitance $C_{b}$ connected to the base terminal of the top transistors. The impedance is calculated as [2]

$$
Z_{\text {emitter }} \approx\left(\frac{1}{g_{m}}\right)\left(1+\frac{C_{b e}}{C_{b}}\right) .
$$

Where $g_{m}$ is the transistor transconductance, and $C_{b e}$ is the base to emitter capacitance.

But for high frequency design, considering the parasitic capacitance effect, the optimum load impedance of the device needs some inductance. The small signal equivalent circuit of Fig. 1 is shown in Fig. 2.

According to Kirchoff's law, the input impedance of the top transistors is calculated as

$$
Z_{e 2} \approx \frac{C_{b}+C_{b e 2}+C_{b} R_{b} / r_{b e 2}}{C_{b} g_{m}}+j \frac{R_{b} \omega^{2} C_{b e 2} C_{b}-1 / r_{b e 2}}{\omega C_{b} g_{m}} .
$$

In Eq. (2), if $R_{b} \omega^{2} C_{b e 2} C_{b}>1 / r_{b e 2}$, the impedance shows some inductive. There are two degrees of freedom to alter the input impedance of the top transistors, and it is easy to obtain an inductive impedance equal to the optimum load impedance of the bottom transistors. To achieve the maximum output power of the PA, the capacitor $C_{b}$ and resistor $R_{b}$ connected 


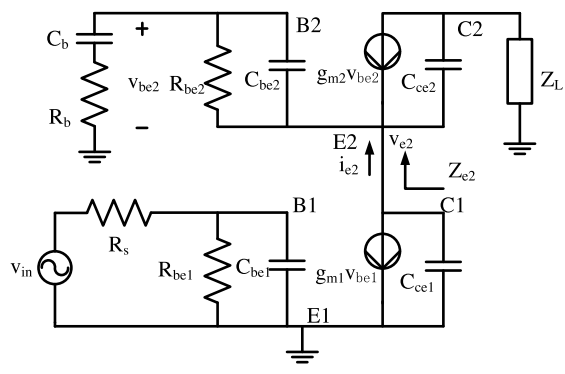

Fig. 2. Small signal equivalent schematic of the dual stacked power amplifier.

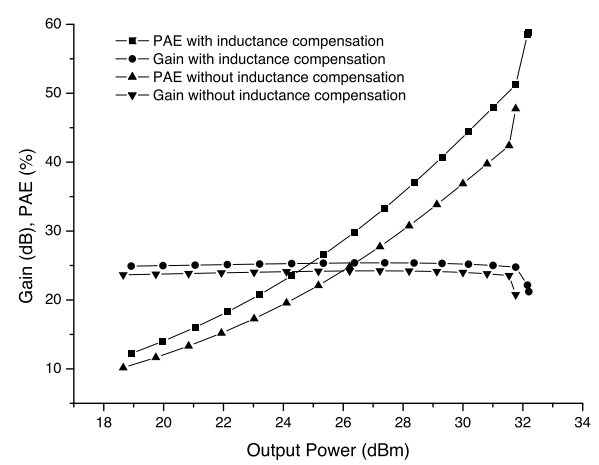

Fig. 3. Simulated gain and PAE versus output power at $2.4 \mathrm{GHz}$.

to the base terminal of the top transistors are properly designed to assure the maximum power transfer to the top transistors. This design suppresses the parasitic capacitance effect, improves PAE and linearity of the power amplifier.

Fig. 3 shows the comparison of simulated PAE between the stacked amplifier with and without inductance compensation. The results show that the PAE can be enhanced by more than $10 \%$ at $1-\mathrm{dB}$ output power with inductance compensation design.

\section{Measurement}

The power amplifiers with and without inductance compensation are fabricated with $2 \mu \mathrm{m} \mathrm{InGaP/GaAs} \mathrm{HBT} \mathrm{technology.} \mathrm{The} \mathrm{die} \mathrm{photo} \mathrm{is} \mathrm{shown} \mathrm{in}$ Fig. 4. And the chip is mounted in the center of a test board with wires

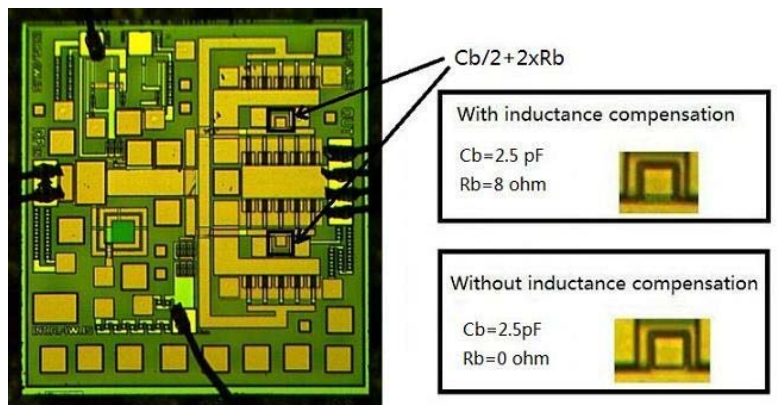

Fig. 4. Die photo of dual stacked power amplifier. 


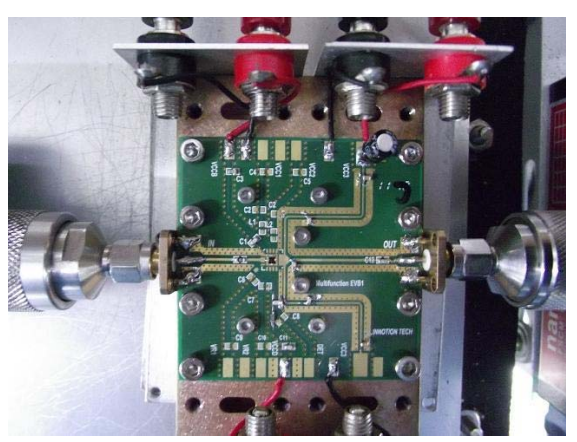

Fig. 5. Test PCB board.

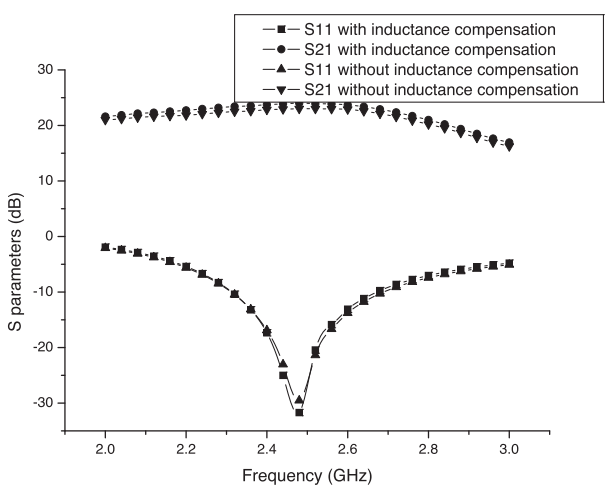

Fig. 6. Measured S-parameters of dual stacked power amplifier.

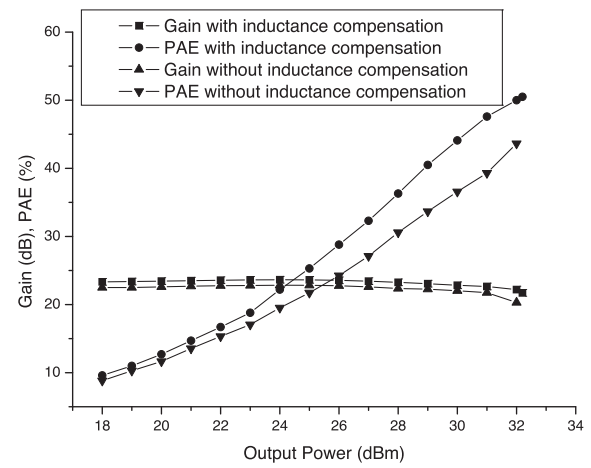

Fig. 7. Measured gain and PAE versus output power at $2.4 \mathrm{GHz}$.

bonded to the board, shown as Fig. 5. The measured small signal gain and return loss are plotted in Fig. 6. The small signal gain is $23.7 \mathrm{~dB}$ at $2.4 \mathrm{GHz}$, and the input return loss is better than $17 \mathrm{~dB}$ from $2.4 \mathrm{GHz}$ to $2.5 \mathrm{GHz}$ with inductance compensation. Fig. 7 shows the measurement results of power gain and PAE as a function of output power at the design frequency of $2.4 \mathrm{GHz}$. The PAE is improved by more than $6 \%$ with inductance compensation at 1-dB output power. With inductance compensation, the 1-dB output power is $32 \mathrm{dBm}$ with $50 \%$ PAE. Fig. 8 shows EVM measured with the 802.11g OFDM/64QAM signal. The EVMs are lower than $3 \%$ up to $26 \mathrm{dBm}$ output power, but increase dramatically beyond this power. And the EVM 


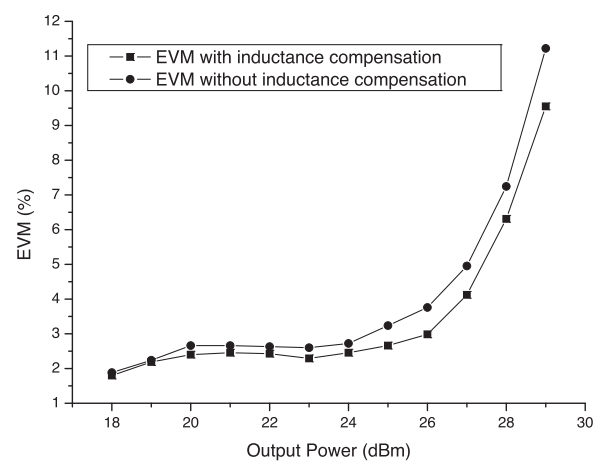

Fig. 8. Measured EVM as a function of output power at $2.4 \mathrm{GHz}$.

is improved by $0.7 \%$ with inductance compensation at $26 \mathrm{dBm}$ output power.

\section{Conclusion}

In this letter, a novel stacked power amplifier structure has been proposed. It is targeted for high frequency application without the need for lossy and bulky inter-matching inductors. The measurement results show that PAE and EVM are improved with this technique. And a power amplifier with this inductance compensation technique is demonstrated to deliver $32 \mathrm{dBm}$ output power with $50 \% \mathrm{PAE}$ at $2.4 \mathrm{GHz}$ with $8 \mathrm{~V}$ voltage supply. The OFDM/64QAM modulation stimulus for the PA test yields $3 \%$ EVM under $26 \mathrm{dBm}$ output power.

\section{Acknowledgments}

The authors would like to thank Professor De Zhang, Nanjing University, China, for the discussions. 